\title{
THE LEVELS OF RELAXIN AND AMINO ACIDS PLAY A CRITICAL ROLE IN WOMEN WITH VARIABLE DEGREE OF PREPAREDNESS FOR LABOUR
}

\author{
Nikolay Shcherbina \\ Department of Obstetrics and Gynecology No. $1^{1}$ \\ Natalia Shelest \\ Department of Obstetrics and Gynecology No. $1^{1}$ \\ ${ }^{1}$ Kharkiv National Medical University \\ 4 Nauky ave., Kharkiv, Ukraine, 61022
}

\begin{abstract}
Underlying biochemical factors that contribute towards the preparedness for labour and ripening of the cervix remain largely unknown. We aimed to characterize metabolic and hormonal determinants that constitute the preparedness for labour in women.

The aim of the research was to study the content of relaxin and amino acids involved in its formation in primiparous pregnant women with various degrees of birth preparedness.

Materials and methods: Prospective study has been conducted on 115 primiparous women at 38-40 weeks of gestation. Biochemical methods were used to analyse relaxin and amino acid levels in pregnant women with varying degree of birth preparedness.

Results: the obtained data from the study indicate importance role of relaxin in the underlying pathogenesis in women with variable degree of preparedness for labour. The results allow to use the levels of relaxin serum as a predictor of the state of the birth canal. Furthermore, the amino acids are known to participate in various critical metabolic processes and play an important role is orchestrating many essential pathways in the body. We present the analysis of amino acids involved in the formation of relaxin in the serum of pregnant women. Our analysis has shown that amino acid imbalance leads to disruption of energy metabolism and blood flow rate, resulting in metabolic, structural and functional changes in the cervix at the onset of labour.

Conclusion: considering the mechanisms of the possible influence of relaxin and amino acids on the cervical ripening, we concluded that correcting amino acid imbalance and normalising relaxin levels should be included in preinduction of labour therapeutic regimen. This would be an important step in improving the perinatal outcomes.
\end{abstract}

Keywords: labor induction, relaxin, cervical ripening, amino acids imbalance.

DOI: $10.21303 / 2504-5679.2021 .001683$

\section{Introduction}

Abnormal labour is a major problem in obstetrics and neonatology, which making increasingly significant contributions to maternal morbidity. The body's birth preparedness is determined by a number of signs, the appearance of which indicates the possibility of a spontaneous labor onset $[1,2]$. With better understanding of normal and abnormal parturition we may be able to understand the critical role of endocrine-immune interaction in the process of labour. The main test of the birth canal maturity is considered to be the morpho-functional assessment of the uterine readiness for childbirth $[3,4]$.

Preparedness for the onset of labour affects the complications that pregnant women, foetus and new-born experience and it remains an important focus of research in modern obstetrics and gynecology $[5,6]$.

Literature review has shown that previous studies have focused largely on the positive and negative pregnancy outcomes in women suffering from gestation complications displayed in their obstetrics and gynaecology medical history [7, 8]. The underlying causes of lack of preparedness for the onset of labour in women in their first pregnancy remains unknown. Considering the medical, social and demographic importance of safe labour in women in their first pregnancy, this study focuses on understanding the pathological criteria of cervix ripening $[9,10]$.

Recent studies have drawn much attention to the hormone relaxin, which is considered as a predictor of pathological labour [11]. 
Relaxin is a polypeptide hormone with a molecular weight of $5-6 \mathrm{kDa}$ belongs to the IGF family of hormones (insulin-like growth factor). It consists of two peptide chains A and B linked by disulphide bridges. The relaxin chain has 22 amino acid residues, and the B chain has 30 amino acid residues. Relaxin is an important participant in many biological processes at all stages of pregnancy. Moreover, both its deficiency and excess can have negative consequences for pregnancy [12, 13].

In obstetrics relaxin is involved in the pathogenesis of abortion [14]. In addition, in the late stages of gestation, relaxin promotes uterine maturity by regulating the sequential processes of collagen degradation and remodelling, causes relaxation of the ligaments of the pubic joint of the pelvic bones, thus carrying out optimal preparation of the maternal organism for physiological childbirth $[15,16]$.

The amino acids involved in the formation of relaxin include: alanine, aspartic, cysteic and glutamic acids, glycine, histidine, tyrosine, and valine. Amino acids, due to their chemical structure (the presence of carboxy and amino groups), participate in the regulation of many reactions, having their own specifics, which determines different direction of their effects. However, the number of studies researching the content of amino acids in the blood of women during pregnancy, especially before labour, is insignificant [17]. Thus, the question of the informativeness of changes in the level of individual amino acids in the body, their importance for the regulation of physiological body preparedness for childbirth, diagnosis, as well as metabolic correction of amino acid imbalance remains practically unresolved [18, 19].

The aim of the research was to study the content of relaxin and amino acids involved in its formation in primiparous pregnant women with various degrees of birth preparedness.

\section{Materials and methods}

All experiments were conducted between 2019-2020 at the clinical bases of the Department of Obstetrics and Gynecology No. 1, Kharkiv National Medical University, Kharkiv, Ukraine.

Prospective study has been conducted on 115 primiparous women at 38-40 weeks of gestation. Depending on the degree of uterine birth preparedness, the examined were divided into two groups. The degree of the uterine birth preparedness was assessed using the E. H. Bishop scale. Group I (control) consisted of 40 (34.8\%) primiparous pregnant women with a mature uterine (9-13 points); Group II (main) included 75 (65.2 \%) women with immature uterine (0-5 points).

The criteria for including patients in the study groups were: the first pregnancy at 38-40 weeks, the absence of severe extragenital pathology, and the absence of late gestosis.

The criteria for excluding from the observation group were: repeated pregnancy and childbirth, pregnancy occurred as a result of assisted reproductive technologies, women who had indications for planned delivery by cesarean section, acute and exacerbation of chronic inflammatory diseases, severe extragenital pathology, and late gestosis.

Ethical approval was obtained from the Bioethics committee of Kharkiv National Medical University (protocol No. 2, 10.06.2020). All participants consented to take part in the study. All experiments involving participants were conducted in accordance with World Medical Association Declaration of Helsinki.

Experimental procedures. The peripheral venous blood was the study material. The content of relaxin in the blood was determined by the enzyme-linked immunosorbent assay using the Human RLN2 ELISA kit (Elabsience, USA).

The content of free amino acids was determined according to the standard method [20] on an LC5001 amino acid analyzer from Biotronic (Germany).

Statistical processing of the obtained data was carried out by the methods of variational analysis using the Student's t-test.

\section{Research results}

Most of the women in the control group were between the ages of 21-25 (40.0\%). The largest number of women aged 31 to 35 years was in the main group (30.7\%). There were $8(20.0 \%)$ and $12(16.0 \%)$ pregnant women at the age of 26-30 years in the control and main group, respectively.

In general, a high frequency of gynecological diseases was revealed in the examined groups. It should be noted that in the group of women with mature uterine (control group), the frequency of 
these diseases was 2.2 times lower than in the main group $(p<0.05)$. The structure of gynecological morbidity was represented mainly by diseases of the cervix, chronic inflammatory diseases of the uterine appendages and abnormal uterine bleeding. 13 (32.5\%) pregnant women in the control group and $15(20.0 \%)$ pregnant women in the main group were gynecologically healthy. The incidence of cervical pathology in the control group was $37.5 \%$ and $46.7 \%$ in the main group. Inflammatory diseases of the genitals were observed in $15.0 \%$ of pregnant women in the control group and in $60.0 \%$ of women in the main group. Abnormal uterine bleeding was twice as common in pregnant women of the main group. Benign diseases of the uterine appendages were found in $5.0 \%$ and $8.0 \%$ in control and main groups, respectively.

Undoubtedly, extragenital pathology had a significant effect on the course of the gestational process, the preparation of the body for childbirth.

When examining the medical records of pregnant women, we paid attention to a high frequency of concomitant extragenital pathology. In the structure of diseases the following prevailed: respiratory diseases in $32.5 \%$ (control group), and $40.0 \%$ (main group) of women; neurocirculatory dystonia in $27.5 \%$ and $29.3 \%$ of pregnant women in the control and main groups; diseases of the urinary system, mainly chronic pyelonephritis in $10.0 \%$ and $24.0 \%$ of pregnant women in the control and main groups; diseases of the gastrointestinal tract in $7.5 \%$ and $21.3 \%$ of women in the control and main groups; diseases of the thyroid gland in $5.0 \%$ and $28.0 \%$ of pregnant women in the control and main groups. Since thyroid hormones increase the sensitivity of the ovaries to gonadotropic hormones and the endometrium to estrogens, together with thyroid hormones deficiency, there occurs estrogen metabolism change and disruption of the process of birth preparedness [21].

The hypertensive type prevailed among the main group patients with neurocirculatory dystonia, which indicated dysregulation of the cardiovascular system. There were 17 (56.7\%) somatically healthy women in the control group and 19 (25.3\%) in the main group. $16(53.3 \%)$ of the examined pregnant women in the control group and $35(46.7 \%)$ in the main group had childhood infections (measles, rubella, chickenpox) which was proved by their medical records.

Estimating the body mass index in pregnant women at the time of admission according to the formula BMI = weight $(\mathrm{kg}) /$ height $^{2}(\mathrm{~m})$. It was found that $5(12.5 \%)$ pregnant women in the control group and $8(10.7 \%)$ women the main group had obesity. A combination of factors such as first childbirth, BMI $\geq 30.1$ can be risk factors for insufficient preparation of a woman's body for childbirth.

Thus, a high frequency of gynecological diseases, extragenital pathology, overweight in women of the study groups, as a manifestation of dysfunction of the central regulatory link, can adversely affect the uterine maturity, and are also regarded as risk factors in assessing the degree of body birth preparedness.

The study of the relaxin concentration showed that women with immature uterine had low values of this hormone $(208.9 \pm 27.6 \mathrm{pg} / \mathrm{ml})$ in the blood serum, compared with similar indicators in the control group $(361.8 \pm 38.4 \mathrm{pg} / \mathrm{ml}), p<0.05$. It can be assumed that the low level of relaxin in the group of pregnant women with immature uterine inhibited the process of cervical dilatation. Considering the mechanisms of the possible influence of relaxin on the state of the birth canal readiness, one can note its ability to change the activity of matrix metalloproteinases that control the structure of glycoproteins in the extracellular matrix $[8,13,15]$.

The analysis of amino acids involved in the formation of relaxin in the blood serum of pregnant women of groups I and II is presented in Table 1.

When analyzing the above data, we noted the increased amount of aspartic acid in the main group. This acid plays an important role in nitrogen metabolism. Glutamic acid, which is involved in energy metabolism, was also detected in an increased amount. Since these amino acids interconnect nitrogen and energy metabolism, a change in their level affects the state of metabolism in cells and tissues.

A 1.7-fold decrease in the content of cysteine was found in the main group of pregnant women in comparison with the control group. This amino acid with metabolic functions is involved in antioxidant processes; it has membrane-protective properties, and can also enhance anabolic processes. 
Table 1

The levels of amino acids in the blood serum of the examined pregnant women $(\mathrm{mg} / \mathrm{l})$

\begin{tabular}{ccc}
\hline \multirow{2}{*}{ Amino acid } & \multicolumn{2}{c}{ Study groups } \\
\cline { 2 - 3 } & Group I (control, $\boldsymbol{n = 4 0 )}$ & Group II (main, $\boldsymbol{n}=\mathbf{7 5})$ \\
\hline Aspartic acid & $14.2 \pm 1.2$ & $18.7 \pm 1.9^{*}$ \\
Glutamic acid & $48.7 \pm 4.8$ & $69.9 \pm 4.1^{*}$ \\
Glycine & $12.3 \pm 1.3$ & $10.2 \pm 1.4^{*}$ \\
Alanin & $25.9 \pm 2.7$ & $26.1 \pm 1.7$ \\
Valine & $22.4 \pm 3.9$ & $21.9 \pm 1.8$ \\
Tyrosine & $12.3 \pm 0.2$ & $13.6 \pm 0.3^{*}$ \\
Histidine & $16.4 \pm 2.4$ & $27.5 \pm 2.3^{*}$ \\
Cysteine & $2.5 \pm 1.3$ & $1.4 \pm 0.8^{*}$
\end{tabular}

Note: $* p<0.05$ - statistical significance of differences between indicators of groups I and II

The histidine content was increased in the main group by $24 \%$. An increase in histidine content inhibits $\mathrm{pH}$-dependent and $\mathrm{pH}$-independent transport of a number of neutral amino acids. In addition, an increase in the amount of histidine may be accompanied by an increase in the production of histamine, which has vasoconstrictor properties and, therefore, impairs blood flow. As a result, tissue hypoxia occurs.

A significant increase in the aromatic amino acid tyrosine in the main group indicates the activity of energy and metabolic processes in the cell membranes. At the same time, the obtained results showed the depletion of the glycine fund, the main sulfur-containing amino acid having neurotransmitter and immunomodulatory effects.

\section{Discussion}

Assessment of cervical ripening and preparedness for labour is instrumental in safe pregnancy and labour. It is therefore important to understand key biological and biochemical determinants that would serve as an effective readout for the assessment of the preparedness for labour.

Previous studies have implicated relaxin as an important factor in predicting delivery in high-risk pregnancies [22]. Furthermore, relaxin has been shown to facilitate cervical ripening and trigger parturition [23]. Of note, exogenous relaxin has also been trialled to treat a number of health conditions including heart failure [24] suggesting that relaxin therapy is safe and may be used in synergy in high risk patients during pregnancy. Our work was focused on understanding the levels of relaxin in women with different preparedness for labour and whether these levels could be used to assess the reediness for the onset of labour. Our data clearly demonstrates that relaxin is an effective tool is assessing the state of the uterus in preparation for labour and therefore serves as an indicator for the preinduction of labour and can potentially be modulated using targeted therapies.

In addition to relaxin levels, the presence of gynecological diseases in our study groups had an effect on gestation process. However, biochemical analysis of the impact of individual pathologies associated with gynecological diseases on the relaxin levels was beyond the scope of this study. Future research could explore whether any secondary health conditions associated with specific gynecological pathologies have an additional effect on relaxin levels.

Current work in our department is focusing on understanding the molecular basis of the effect of relaxin on the host cells. Understanding the intracellular signalling cascade that relaxin triggers in the uterus at different stages during pregnancy will illuminate further the role of this multifunctional molecule in determining pregnancy outcomes.

Amino acids are essential building blocks of life. The role of amino acids in protein synthesis, metabolism, signalling, immune response has extensively been covered in literature. Previous studies have identified the role of several amino acids in pregnancy outcomes. For example, the 
development of pathologies, such as pre-eclampsia and placental dysfunction, is often accompanied by changes in the amino acids levels, which in turn affects the course of effective adaptation to pregnancy $[25,26]$. Here we asked a question whether amino acids essential for the synthesis of relaxin may be involved in determining the outcome of pregnancy. Our work demonstrates that limited or excessive levels of cystine and aspartic acid, histidine and tyrosine respectively were linked to a number of negative outcomes in women with variable degree of labour preparedness.

Study limitations. The study involved a small number of patients. Future studies will benefit from expanding the number of patients in both study and control groups.

The work presented here did not conduct follow up post-partum analysis of women involved in the study.

Prospects for further research. Future work will focus on understanding whether there is a link between the diet, which is the direct source of the amino acids, and amino acid levels in women with different preparedness for labour. Furthermore, we would like to investigate the expression levels of different core enzymes involved in amino acid metabolism at the genetic level. This will reveal the amino acid requirements of the host at different stages of gestation and serve as an atlas of amino acid metabolism during pregnancy.

Future work serves an important basis for designing novel therapeutic interventions to target relaxin and amino acids levels to prevent negative perinatal outcomes.

\section{Conclusions}

1. A $43 \%$ drop in relaxin levels was observed in women with immature uterine compared to control group. This implicates relaxin in the pathogenesis in women with variable degree of preparedness for labour. This observation allows to consider the serum levels of relaxin as a predictor of the biological birth preparedness of the body.

2. We noted a significant amino acid imbalance in the studied patients, in particular in amino acids associates with production of relaxin. Since amino acids play an important role in homeostasis including energy metabolism and blood flow rate, our results suggesting that disruption of amino acid balance may in turn lead to structural and functional changes in the cervix.

3. The analysis presented here suggests the need to use agents that modulate relaxin levels during the pre-induction labour and correct amino acid imbalance during preparation for labour.

\section{Conflicts of interest}

The authors declare that they have no conflicts of interest.

\section{References}

[1] Shah, J., Baxi, B. (2016). Identification of biomarkers for prediction of preterm delivery. Journal of Medical Society, 30 (1), 3. doi: http://doi.org/10.4103/0972-4958.175790

[2] Golightly, E., Jabbour, H. N., Norman, J. E. (2011). Endocrine immune interactions in human parturition. Molecular and Cellular Endocrinology, 335 (1), 52-59. doi: http://doi.org/10.1016/j.mce.2010.08.005

[3] Shah, J., Baxi, B. (2016). Identification of biomarkers for prediction of preterm delivery. Journal of Medical Society, 30 (1), 3. doi: http://doi.org/10.4103/0972-4958.175790

[4] Iliodromiti, Z., Antonakopoulos, N., Sifakis, S., Tsikouras, P., Daniilidis, A., Dafopoulos, K. et. al. (2012). Endocrine, paracrine, and autocrine placental mediators in labor. Hormones, 11 (4), 397-409. doi: http://doi.org/10.14310/horm.2002.1371

[5] Goldberg, A. E. (2020). Cervical Ripening. Available at: https://emedicine.medscape.com/article/263311-overview

[6] Kanayama, N. (2019). Cervical Changes 1: Morphological and Biochemical Changes. Comprehensive Gynecology and Obstetrics, 61-75. doi: http://doi.org/10.1007/978-981-13-9875-9_6

[7] Lizewska, B., Teul, J., Kuc, P., Lemancewicz, A., Charkiewicz, K., Goscik, J. et. al. (2018). Maternal Plasma Metabolomic Profiles in Spontaneous Preterm Birth: Preliminary Results. Mediators of Inflammation, 2018, 1-13. doi: http://doi.org/ 10.1155/2018/9362820

[8] Ludmir, J., Sehdev, H. M. (2000). Anatomy and Physiology of the Uterine Cervix. Clinical Obstetrics and Gynecology, 43 (3), 433-439. doi: http://doi.org/10.1097/00003081-200009000-00003

[9] Maul, H., Mackay, L., Garfield, R. E. (2006). Cervical Ripening: Biochemical, Molecular, and Clinical Considerations. Clinical Obstetrics and Gynecology, 49 (3), 551-563. doi: http://doi.org/10.1097/00003081-200609000-00015 
[10] Word, R., Li, X.-H., Hnat, M., Carrick, K. (2007). Dynamics of Cervical Remodeling during Pregnancy and Parturition: Mechanisms and Current Concepts. Seminars in Reproductive Medicine, 25 (1), 69-79. doi: http://doi.org/10.1055/s-2006-956777

[11] Goldsmith, L. T., Weiss, G. (2009). Relaxin in Human Pregnancy. Annals of the New York Academy of Sciences, 1160 (1), 130-135. doi: http://doi.org/10.1111/j.1749-6632.2008.03800.x

[12] Vogel, I., Thorsen, P., Hundborg, H. H., Uldbjerg, N. (2006). Prediction of preterm delivery using changes in serum relaxin in low risk pregnancies. European Journal of Obstetrics \& Gynecology and Reproductive Biology, 128 (1-2), 113-118. doi: http:// doi.org/10.1016/j.ejogrb.2005.11.002

[13] Eppel, W., Kucera, E., Bieglmayer, C. (1999). Relationship of serum levels of endogenous relaxin to cervical size in the second trimester and to cervical ripening at term. BJOG: An International Journal of Obstetrics and Gynaecology, 106 (9), $917-923$. doi: http://doi.org/10.1111/j.1471-0528.1999.tb08430.x

[14] Hou, Q., Jiang, C., Huang, Y., Ye, J., Yang, X. (2018). Is maternal serum relaxin associated with preterm delivery in Chinese pregnant women? A meta-analysis. The Journal of Maternal-Fetal \& Neonatal Medicine, 32 (20), 3357-3366. doi: http:// doi.org/10.1080/14767058.2018.1463983

[15] Sherwood, O. D. (2004). Relaxin's Physiological Roles and Other Diverse Actions. Endocrine Reviews, 25 (2), $205-234$. doi: http://doi.org/10.1210/er.2003-0013

[16] Simpson, K. R. (2020). Cervical Ripening and Labor Induction and Augmentation, 5th Edition. Nursing for Women's Health, 24 (4), S1-S41. doi: http://doi.org/10.1016/j.nwh.2020.04.005

[17] Schulpis, K. H., Vlachos, G. D., Papakonstantinou, E. D., Karikas, G. A., Vlachos, D. G., Roma, E. et. al. (2009). Maternal-neonatal amino acid blood levels in relation to the mode of delivery. Acta Obstetricia et Gynecologica Scandinavica, 88 (1), 71-76. doi: http://doi.org/10.1080/00016340802578098

[18] Osmers, R. G. W., Adelmann-Grill, B. C., Rath, W., Stuhlsatz, H. W., Tschesche, H., Kuhn, W. (1995). Biochemical Events in Cervical Ripening Dilatation during Pregnancy and Parturition. Journal of Obstetrics and Gynaecology, 21 (2), $185-194$. doi: http://doi.org/10.1111/j.1447-0756.1995.tb01092.x

[19] Yellon, S. M. (2020). Immunobiology of Cervix Ripening. Frontiers in Immunology, 10. doi: http://doi.org/10.3389/ fimmu.2019.03156

[20] Benson, J. V., Patterson, J. A.; Ovchinnikov, Yu. A. (Ed.) (1974). Hromatograficheskiy analiz aminokislot i peptidov na sfericheskih smolah i ego primenenie v biohimii i meditsine. Novyie metodyi analiza aminokislot, peptidov i belkov. Moscow: Mir, 9-84

[21] Vannuccini, S., Bocchi, C., Severi, F. M., Challis, J. R., Petraglia, F. (2016). Endocrinology of human parturition. Annales d'Endocrinologie, 77 (2), 105-113. doi: http://doi.org/10.1016/j.ando.2016.04.025

[22] Vogel, I., Glavind-Kristensen, M., Thorsen, P., Armbruster, F. P., Uldbjerg, N. (2002). S-relaxin as a predictor of preterm delivery in women with symptoms of preterm labour. BJOG: An International Journal of Obstetrics and Gynaecology, 109 (9), 977-982. doi: http://doi.org/10.1111/j.1471-0528.2002.01187.x

[23] Evans, M. I., Dougan, M.-B., Moawad, A. H., Evans, W. J., Bryant-Greenwood, G. D., Greenwood, F. C. (1983). Ripening of the human cervix with porcine ovarian relaxin. American Journal of Obstetrics and Gynecology, 147 (4), 410-414. doi: http:// doi.org/10.1016/s0002-9378(16)32236-0

[24] Teerlink, J. R., Metra, M., Felker, G. M., Ponikowski, P., Voors, A. A., Weatherley, B. D. et. al. (2009). Relaxin for the treatment of patients with acute heart failure (Pre-RELAX-AHF): a multicentre, randomised, placebo-controlled, parallel-group, dose-finding phase IIb study. The Lancet, 373 (9673), 1429-1439. doi: http://doi.org/10.1016/s0140-6736(09)60622-x

[25] Evans, R., Powers, R., Ness, R., Cropcho, L., Daftary, A., Harger, G. et. al. (2003). Maternal and fetal amino acid concentrations and fetal outcomes during pre-eclampsia. Reproduction, 125 (6), 785-790. doi: http://doi.org/10.1530/rep.0.1250785

[26] Cetin, I., de Santis, M. S. N., Taricco, E., Radaelli, T., Teng, C., Ronzoni, S. et. al. (2005). Maternal and fetal amino acid concentrations in normal pregnancies and in pregnancies with gestational diabetes mellitus. American Journal of Obstetrics and Gynecology, 192 (2), 610-617. doi: http://doi.org/10.1016/j.ajog.2004.08.011

Received date 02.12.2020

(C) The Author(s) 2021

Accepted date 05.02.2021

Published date 31.03.2021

This is an open access article under the CC BY license (http://creativecommons.org/licenses/by/4.0).

How to cite: Shcherbina, N., Shelest, N. (2021). The levels of relaxin and amino acids play a critical role in women with variable degree of preparedness for labour. EUREKA: Health Sciences, 2, 3-8. doi: http://doi.org/10.21303/2504-5679.2021.001683 